\title{
Analyzing Social Network Images with Deep Learning Models to Fight Zika Virus
}

\author{
Pedro H. Barros ${ }^{1}$, Bruno G. C. Lima ${ }^{1}$, Felipe C. Crispim ${ }^{1}$, Tiago Vieira ${ }^{1}$, \\ Paolo Missier ${ }^{2}$, and Baldoino Fonseca ${ }^{1}$ \\ 1 Federal University of Alagoas, Maceió, Brazil, \\ \{pedro_h_nr, bgcl,fcc, tvieira, baldoino\}@ic.ufal.br, \\ http://www.ic.ufal.br/ \\ 2 Newcastle University, Newcastle, United Kingdom, \\ paolo.missier@newcastle.ac.uk, \\ http://www.ncl.ac.uk/
}

\begin{abstract}
Zika and Dengue are viral diseases transmitted by infected mosquitoes (Aedes aegypti) found in warm, humid environments. Mining data from social networks helps to find locations with highest density of reported cases. Differently from approaches that analyze text from social networks, we present a new strategy analyzing Instagram images. We use two customized Deep Neural Networks. The first detects objects commonly used for mosquito reproduction with $85 \%$ precision. The second differentiates mosquitoes as Culex or Aedes aegypti with $82.5 \%$ accuracy. Results indicate that both networks can improve the effectiveness of current social network mining strategies such as the VazaZika project.
\end{abstract}

Keywords: Deep Neural Networks, Zika, Aedes aegypti, Social Networks

\section{Introduction}

Zika has become a big problem for health organizations. Among worst disease implications, we highlight microcephaly [12] in newborns of contaminated mothers. Transmitted by the Aedes aegypti mosquito, reported contaminations of Zika are mainly present in countries with warm and humid weather. To pinpoint Aedes aegypti breeding sites, one mines data from social networks. Based on user's posts, authorities may retrieve information such as the number of reported cases and their location. VazaZika ${ }^{3}$ [11] is a platform where users may report cases, resulting in a geographical map containing inferred mosquitoes concentration.

We use pictures from Instagram and two Deep Neural Networks. One detects objects commonly used by mosquitoes for egg deposition (tires, bottles and jars). The other classifies whether a mosquito is a Culex (common) or Aedes aegypti (vector of Zika and other diseases). We argue that our solution, attached to the VazaZika platform, improves the effectiveness of health agencies actions by

3 http://vazadengue.inf .puc-rio.br/ 
pinpointing relevant loci. To the extent of our knowledge, no work with this purpose has been presented so far.

We aim at answering the following Research Questions: (RQ1): How effective is our deep learning model for detecting objects associated with mosquito proliferation? (RQ2): How accurate is our mosquito classification model? (RQ3): What is the classification performance when applied to pictures from Instagram?

Our contribution is twofold. First, we collected and annotated an image database comprising: (1) Culex and Aedes aegypti mosquitoes and; (2) tires, empty bottles and plant pots - common mosquito breeding sites. Second, we trained deep models which performed well when tested on pictures from Instagram.

\section{Related work}

Hay et al. [7] used sophisticated airborne and satellite-sensor technology, often unavailable alternatives to poor nations due to high costs. Wang et al. [16] used feature extraction and classification of high resolution close-up images, differently from our approach which deals with uncontrolled images. Jahangir et al. [1] studied insect's characteristics for species classification. Fuchida et al., classified insects as whether mosquito or another bug [5]. Their approach differs from ours since we classify a mosquito between two genera: Aedes and Culex. SVM was also used in [4] to classify species and genus of fruit flies and mosquitoes using only images of their wings. In [8] authors generate a Wavelet representation of mosquitoe's sounds, passing it as input to a Convolutional Neural Network $(\mathrm{CNN})$ classifier. Authors in [14] propose a novel method based on CNN for mosquito larva classification, using Alexnet architecture for the deep model. Dong et al. [2] evaluated Deep Convolutional Neural Network to identify cells infected with malaria. Mehra et al. [10] collected data from multiples sources, combining RGB and thermal images in order to detect the presence of water puddles through an ensemble of Bayesian. Neural networks have also been used for object detection tasks, for example, detecting vehicle [9], pedestrians [6] and faces [15].

We emphasize that, to the extent of our knowledge, no work has been presented so far with the specific goal of using Deep Learning to analyze images from social networks to fight proliferation of mosquito transmitted diseases. Therefore, we present no quantitative comparison with previous works.

\section{Methodology}

From ImageNet ${ }^{4}$ and Google Images, we firstly gathered pictures using the following keywords: (i) aedes aegypti; (ii) culex; (iii) soda bottle; (iv) car tire $^{5}$; (v) flowerpot. Secondly, we collected mosquito pictures from Instagram in order to test the classification model and obtain real performance metrics.

\footnotetext{
${ }^{4}$ www.image-net.org

${ }^{5}$ Particularly suited to mosquitoes for providing black camouflage.
} 


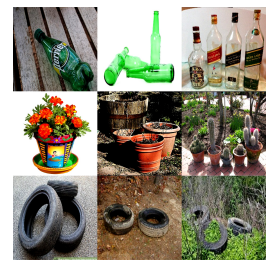

(a)

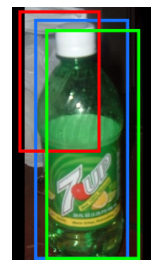

(b)

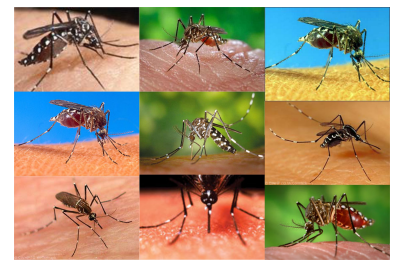

(c)

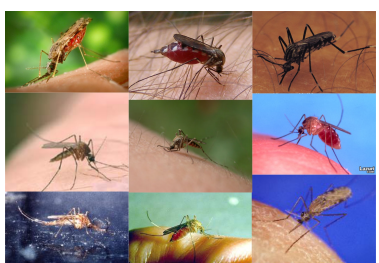

(d)

Fig. 1. Dataset samples. (a) Bottles, recipient and tires. (b) True-positive (green), false-positive (red) and ground-truth (blue). (c) Aedes. (d) Culex.

For the object detection dataset (Fig. 1-(a)), we used 170 images with tires, 167 with bottles and 182 with flower pots (85\% of them for training and $15 \%$ for testing). We used TensorFlow Object Detection API with the Faster RCNN Resnet [13] model and following augmentation options: (i) random horizontal flip; (ii) random vertical flip; (iii) random 90 deg rotation.

The accuracy metric is the Intersection Over Union (IOU) (PASCAL Visual Object Classes Challenge 2007 [3]). For each training step, we evaluated: Average Precision per class $A P$ and Mean Average Precision $m A P$ for all classes. $I O U$ ratio for each bounding box (BB) must exceed a threshold of 0.5 to be considered a "true positive", meaning a "false positive" otherwise. Fig. 1-(b) shows an example of a true and a false positive.

We also gathered 226 images of Aedes and 322 Culex (Fig. 1-(c) and (d), respectivelly). All images were thoroughly verified by experts from local authority in Zoonoses Control Center (ZCC). We also used data augmentation: Random scaling, Rotation in 3 directions $\left(90^{\circ}, 180^{\circ}\right.$ and $\left.270^{\circ}\right)$ and random flip (horizontal and vertical). The final dataset is based on 80 original images for testing (15\% of total) and was increased to 3804 by data augmentation. Finally, we collected 60 pictures of mosquitoes from Instagram to test the trained model, as presented in Fig. 2.

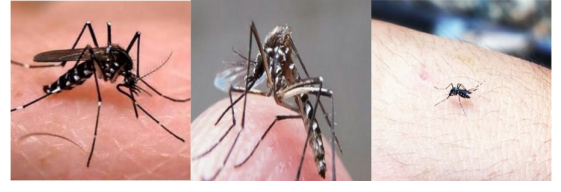

(a)
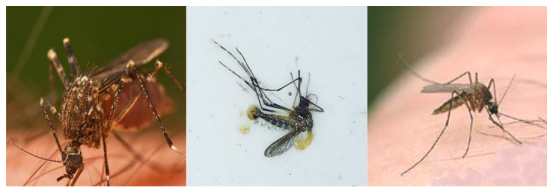

(b)

Fig. 2. Example images belonging to (a) Aedes class, and (b) Culex category posted on Instagram. 


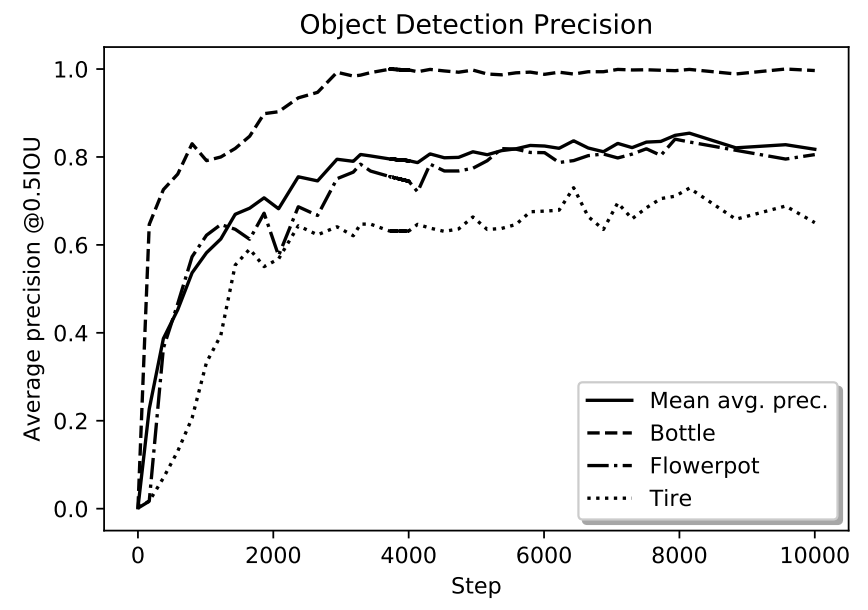

Fig. 3. Average Precision per step index.

\section{Results}

RQ1. Average detection Precision (AP) is presented in Fig. 3. On the best checkpoint, APs achieved 99\%, $84 \%$ and $73 \%$, for bottle, flowerpot and tire, respectively (Mean Average Precision, $\mathrm{mAP}=85 \%$ ).

RQ2. We proposed the following model: (i) Input image is resized to $225 \times$ 225. (ii) Rectifier Linear Units is used as activation function. (iii) Training batch with a size of 32. (iv) 6-fold cross-validation. (v) Random drop-out was used as an attempt to reduce overfitting and improve the network robustness to unseen data. This topology provided a training accuracy of $82.5 \%$.

RQ3. Finally, the model trained over the mosquito dataset was tested on pictures collected from Instagram. Since users do not often post mosquito photographs, it was difficult to gather many images (we found 78 images, but 18 of them were present in our training dataset and were excluded). Hence, we applied the classification model onto 60 images and the results are shown in Fig. 4 (Receiver Operating Characteristic curve and Confusion Matrix).

\section{Discussion}

Results are shown in Fig. 3. Class "bottle" performs best (99\%) since pictures were reasonably well behaved, with few or no occlusions at all. The "flowerpot" class, presenting many images with partial occlusion caused by leaves, flowers and garden utilities, followed with $84 \%$ precision. "Tires" presented worst precision $(73 \%)$ likely due to the large variety of positions (often with big stocks of tires) and occlusion. Model performed satisfactorily overall, achieving a mAP of $85 \%$. 


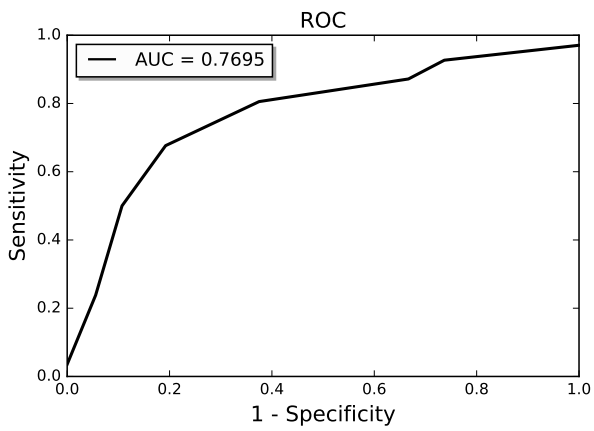

(a)

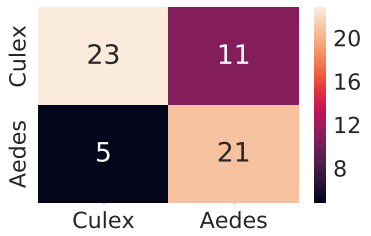

(b)

Fig. 4. Results from applying the mosquito classification model to real images collected from Instagram. (a) Receiver Operating Characteristic curve showing an Area Under Curve of 0.77 . (b) Confusion matrix.

For the classification problem, a Deep Convolutional Neural Network is capable of achieving good training results $(82.5 \%)$ on a challenging dataset using only supervised learning. Testing the model on very few images (60) collected from Instagram provided $73.33 \%$ classification accuracy. This reduction might be expected due to the small amount of training and test image (low generalization capabilities of the network) and due to the variety (scale and rotation) of images in the test set (cf. Fig. 2). In some images the mosquito is very small and sometimes squashed dead. A more comprehensive analysis is to be pursued by using more pictures. Testing different network topologies (auto-encoders) might improve results.

\section{Conclusion}

We presented two Deep Learning Models aimed at processing images and extracting information associated with mosquito activity. To train the models we collected many images from Google Images and Image-Net. Even tough no direct comparison is possible with previous works, training performances were high for both models ( $85 \%$ for detection and $82.5 \%$ for classification) and we tested the classification model on real pictures posted on Instagram (73\% accuracy). More comprehensive experiments are being executed using the VazaZika platform and results will be eventually reported.

\section{Acknowledgments}

The authors would like to thank: (1) National Council for Scientific and Technological Development (CNPq, grant 447336/2014-2). (2) Deep Learning program provided by the Nervana Academy (Intel ${ }^{\circledR}$ ). (3) FAPEAL grant 60030 $1201 / 2016$. 


\section{References}

1. Alam S.M, J., Guoqing, H., Chen, C.: Characteristics analysis and detection algorithm of mosquitoes. TELKOMNIKA Indonesian Journal of Electrical Engineering 17, 5368-5378 (Dec 2013)

2. Dong, Y., Jiang, Z., Shen, H., Pan, W.D., Williams, L.A., Reddy, V.V.B., Benjamin, W.H., Bryan, A.W.: Evaluations of deep convolutional neural networks for automatic identification of malaria infected cells. In: 2017 IEEE EMBS International Conference on Biomedical Health Informatics (BHI). pp. 101-104 (feb 2017)

3. Everingham, M., Gool, L.V., Williams, C.K.I., Winn, J., Zisserman, A.: The pascal visual object classes (voc) challenge. International Journal of Computer Vision 88(2), 303338 (Sep 2009)

4. Favret, C., Sieracki, J.M.: Machine vision automated species identification scaled towards production levels. Systematic Entomology 41(1), 133143 (2015)

5. Fuchida, M., Pathmakumar, T., Mohan, R., Tan, N., Nakamura, A.: Vision-based perception and classification of mosquitoes using support vector machine. Applied Sciences 7(1), 51 (Jan 2017)

6. Geronimo, D., Lopez, A.M., Sappa, A.D., Graf, T.: Survey of pedestrian detection for advanced driver assistance systems. IEEE Transactions on Pattern Analysis and Machine Intelligence 32(7), 1239-1258 (July 2010)

7. Hay, S., Snow, R., Rogers, D.: From predicting mosquito habitat to malaria seasons using remotely sensed data: Practice, problems and perspectives. Parasitology Today 14(8), 306 - 313 (1998), http://www.sciencedirect.com/science/article/ $\mathrm{pii/S016947589801285 \textrm {X }}$

8. Kiskin, I., Orozco, B.P., Windebank, T., Zilli, D., Sinka, M., Willis, K., Roberts, S.: Mosquito Detection with Neural Networks: The Buzz of Deep Learning (2017)

9. Manana, M., Tu, C., Owolawi, P.A.: A survey on vehicle detection based on convolution neural networks. In: 2017 3rd IEEE International Conference on Computer and Communications (ICCC). pp. 1751-1755 (Dec 2017)

10. Mehra, M., Bagri, A., Jiang, X., Ortiz, J.: Image analysis for identifying mosquito breeding grounds. 2016 IEEE International Conference on Sensing, Communication and Networking (SECON Workshops) (2016)

11. Missier, P., McClean, C., Carlton, J., Cedrim, D., Sousa, L., Garcia, A., Plastino, A., Romanovsky, A.: Recruiting from the network: discovering Twitter users who can help combat Zika epidemics (2017)

12. Rasmussen, S., J Jamieson, D., Honein, M., R Petersen, L.: Zika Virus and Birth Defects - Reviewing the Evidence for Causality. The New England journal of medicine 374 (2016)

13. Ren, S., He, K., Girshick, R., Sun, J.: Faster r-cnn: Towards real-time object detection with region proposal networks. IEEE Transactions on Pattern Analysis and Machine Intelligence 39(6), 11371149 (Jan 2017)

14. Sanchez-Ortiz, A., Fierro-Radilla, A., Arista-Jalife, A., Cedillo-Hernandez, M., Nakano-Miyatake, M., Robles-Camarillo, D., Cuatepotzo-Jimenez, V.: Mosquito Larva Classification Method Based on Convolutional Neural Networks. Univ Americas Puebla, Dept Comp Elect \& Mechatron; IEEE (2017), 27th International Conference on Electronics, Communications and Computers (CONIELECOMP)

15. Srivastava, A., Mane, S., Shah, A., Shrivastava, N., Thakare, B.: A survey of face detection algorithms. In: 2017 International Conference on Inventive Systems and Control (ICISC). pp. 1-4 (Jan 2017)

16. Wang, J., Lin, C., Ji, L., Liang, A.: A new automatic identification system of insect images at the order level. Know.-Based Syst. 33, 102-110 (Sep 2012) 